\title{
Bronchial Carcinoid Associated with Atopic Cough in a 31-year-old Male
}

Johsuke Hara $^{1 *}$, Tamami Sakai ${ }^{1}$, Taro Yoneda ${ }^{1}$, HayatoKoba ${ }^{1}$, Keigo Saeki $^{2}$, Mayuko Tani ${ }^{2}$, Noriyuki Ohkura ${ }^{1}$, Takashi Sone ${ }^{1}$, Hideharu Kimura ${ }^{1}$, Yoshihisa Ishiura $^{3}$, Kazuo Kasahara ${ }^{1}$ and Masaki Fujimura ${ }^{4}$

${ }^{1}$ Respiratory Medicine, Kanazawa University Faculty of Medicine, Institute of Medical, Pharmaceutical and Health Sciences, Kanazawa, Ishikawa, Japan

${ }^{2}$ Internal Medicine, Keiju Medical Center, Nanao, Ishikawa, Japan

${ }^{3}$ Respiratory Medicine, Toyama City Hospital, Toyama, Toyama, Japan

${ }^{4}$ Respiratory Medicine, National Hospital Organization Nanao Hospital, Nanao, Ishikawa, Japan

*Corresponding author: Johsuke Hara, Respiratory Medicine, Kanazawa University Faculty of Medicine, Institute of Medical, Pharmaceutical and Health Sciences, Kanazawa, Ishikawa, Japan, Tel: +81-76-265-2000; E-mail: hara0728@gmail.com

Rec date: April 26, 2016 Acc date: May 25, 2016 Pub date: May 31, 2016

Copyright: (C) 2016 Hara J, et al. This is an open-access article distributed under the terms of the Creative Commons Attribution License, which permits unrestricted use, distribution, and reproduction in any medium, provided the original author and source are credited.

\section{Introduction}

In the Hokuriku region of Japan, atopic cough (AC), cough variant asthma (CVA), and sinobronchial syndrome (SBS) are the major causes of chronic cough. However, airway malignant tumors cause persistent cough, and missed or delayed diagnosis of malignancy may result in serious consequences. We report the case of a 31-year-old man who was finally diagnosed with bronchial carcinoid and AC after suffering from a chronic non-productive cough for 6 months.

Keywords: Chronic cough; Atopic cough; Bronchial carcinoid

\section{Case Report}

A 31-year-old man, working as a fisherman, visited his family doctor after a 6-month history of non-productive cough. He also reported a few episodes of yellow sputum and post nasal drip within a month, which spontaneously resolved without treatment.

This non-productive cough worsened at night and was accompanied by itching in the throat. His cough appeared to resolve for brief periods following symptomatic treatment but then returned.

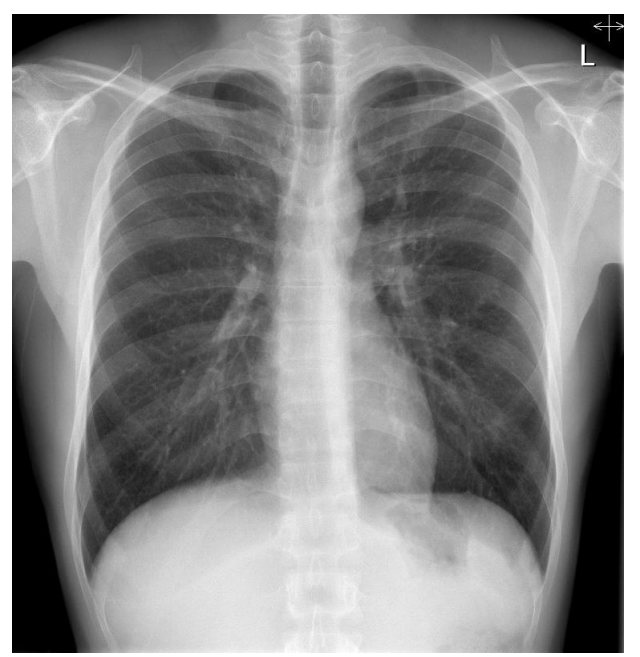

Figure 1: Chest X-ray showing no abnormal findings.

He was referred to our hospital for further treatment. He was a current smoker. His past medical history included perennial and seasonal allergic rhinitis and chronic sinusitis as a child. There was no history of either chronic cough and bronchial asthma or angiotensinconverting enzyme (ACE) inhibitor use. His family history was not clinically significant.

At the initial visit, his vital signs were stable, with a body temperature of $36.8^{\circ} \mathrm{C}$ and hemoglobin oxygen saturation of $98 \%$. Auscultation of the chest was normal. Chest and sinus X-rays were normal (Figure 1).

His peripheral WBC count was $6,200 / \mu$, with $55.6 \%$ neutrophils and $1.1 \%$ eosinophils. Serum C-reactive protein level was $0.0 \mathrm{mg} / \mathrm{dl}$. Total serum IgE level was $143 \mathrm{IU} / \mathrm{mL}$, and antigen-specific IgE against house dust mite (Dermatophagoides farina), cedar pollen, ragweed pollen, timothy pollen, and Anthoxanthum odoratum pollen were positive.

The pulmonary function tests demonstrated no evidence of restrictive or obstructive ventilatory impairments, and no bronchial reversibility (\%FVC, $118.3 \%$; \%FEV1, 117.8\%; FEV1/FVC ratio, $91.1 \%$; bronchial reversibility, $140 \mathrm{~mL}, 3.3 \%)$. Sputum for cytology and detection of pathogens could not been obtained.

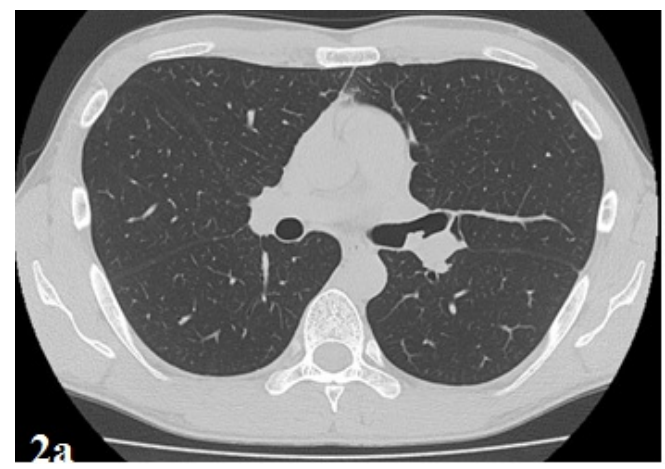

Figure 2a: Chest computed tomography axial and coronal.

His cough was resistant to the bronchodilator therapy $(40 \mu \mathrm{g} /$ day oral clenbuterol for 10 days). Next, he was administered two puffs per day of $200 \mu \mathrm{g}$ fluticasone propionate inhaler, which is an inhaled corticosteroid, and azelastine, which is a histamine $\mathrm{H} 1$ antagonist. After initiating inhaled corticosteroids, his cough almost but not completely resolved. Therefore, chest computed tomography (CT) was performed, which revealed an endobronchial tumor in the left main bronchus (Figures 2a and 2b). 
Citation: Johsuke H, Tamami S, Taro Y, HayatoKoba, Keigo S, et al. (2016) Bronchial Carcinoid Associated with Atopic Cough in a 31-year-old Male. Gen Med (Los Angeles) 4: 244. doi:10.4172/2327-5146.1000244

Page 2 of 4

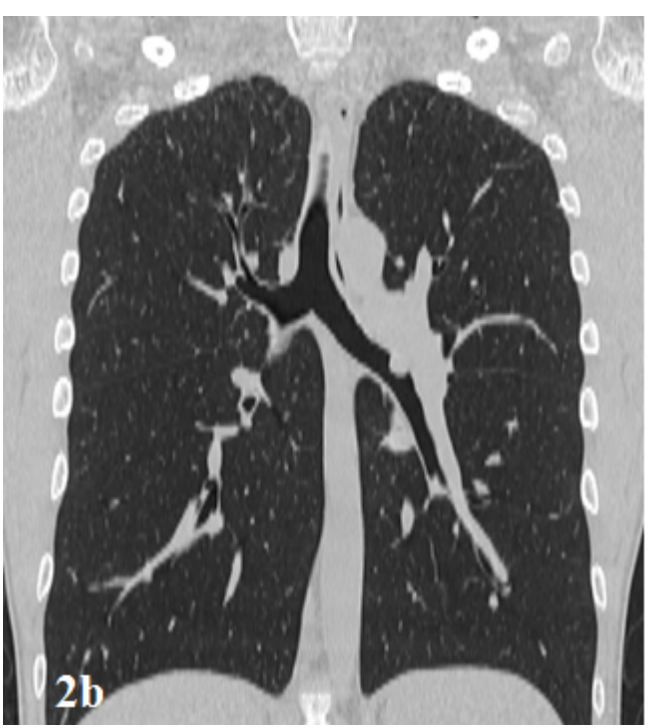

Figure 2b: Images in the lung window showing an endobronchial mass in the left main bronchus.

Positron emission tomography (PET) with [18F] fluoro-2deoxyglucose and CT revealed weak uptake at the endobronchial tumor, with a maximum standardized uptake value of 2.0. There was no evidence of abnormal uptake at the mediastinal lymph nodes or other extrathoracic organs. Flexible bronchoscopy revealed a highly vascularized endobronchial tumor (Figure 3).

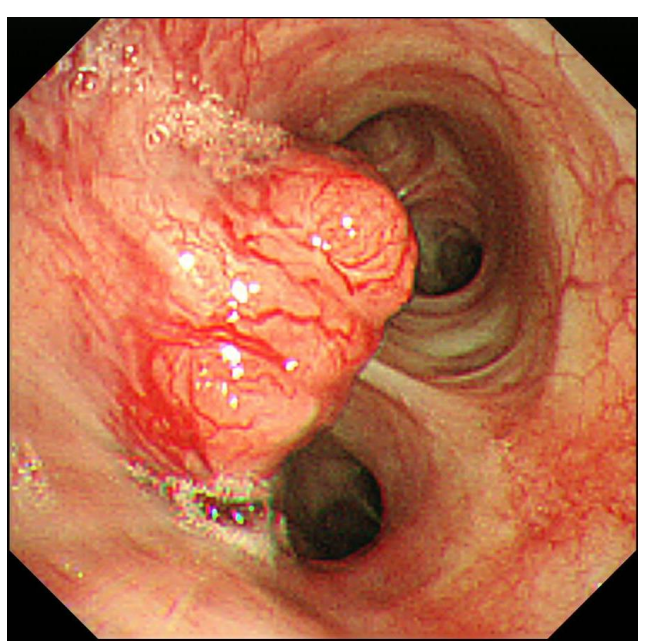

Figure 3: Flexible bronchoscopy showing a highly vascularized endobronchial tumor in the left main bronchus.

To reduce procedure-related hemorrhage, a hot biopsy was performed. The biopsy specimen showed that the tumor cells had round to oval nuclei and the eosinophilic cytoplasm formed a focal and trabecular pattern.

No mitosis or necrosis was present. Tumor cell polymorphism was hardly observed after H\&E staining (Figures $4 \mathrm{a}$ and $4 \mathrm{~b}$ ).

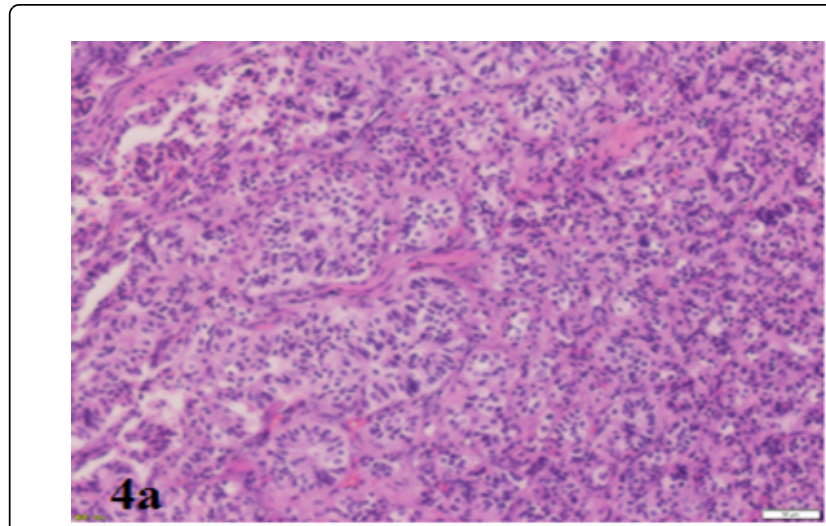

Figure 4a: The biopsy specimen showing proliferation of uniformly oval tumor cells forming focal structures.

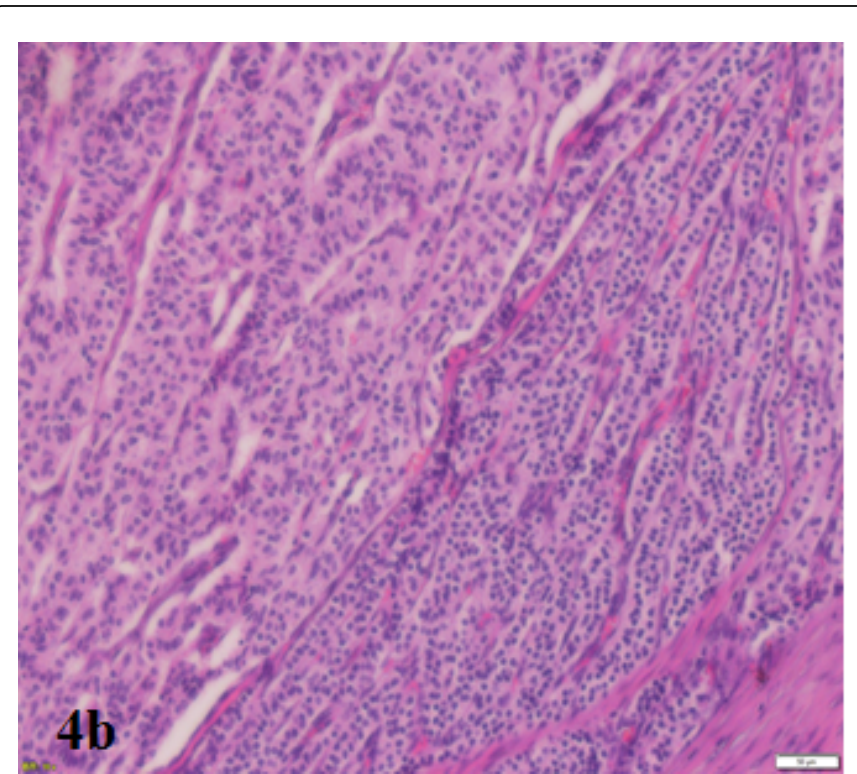

Figure 4b: The biopsy specimen showing proliferation of uniformly oval tumor cells forming trabecular structures.

The tumor presented positive immunoreactivity for chromograninA (Figure 4c), synaptophysin (Figure 4d).

Typical carcinoid was the final clinical diagnosis. He successfully underwent resection of the tumor and left main bronchus and subsequently bronchoplasty. There was no metastasis in the seven dissected mediastinal lymph nodes. The final pathological examination of the surgical specimen confirmed complete resection of the endobronchial-typical carcinoid from the left main bronchus. There were no complications during the postoperative period, and the patient was discharged from the hospital 10 days after the operation. The histamine $\mathrm{H} 1$ antagonist and inhaled corticosteroids were discontinued after several days of complete resolution of his nonproductive dry cough. Five years after surgical treatment, the patient was well, had no cough, and was asymptomatic with no signs of carcinoid recurrence. 


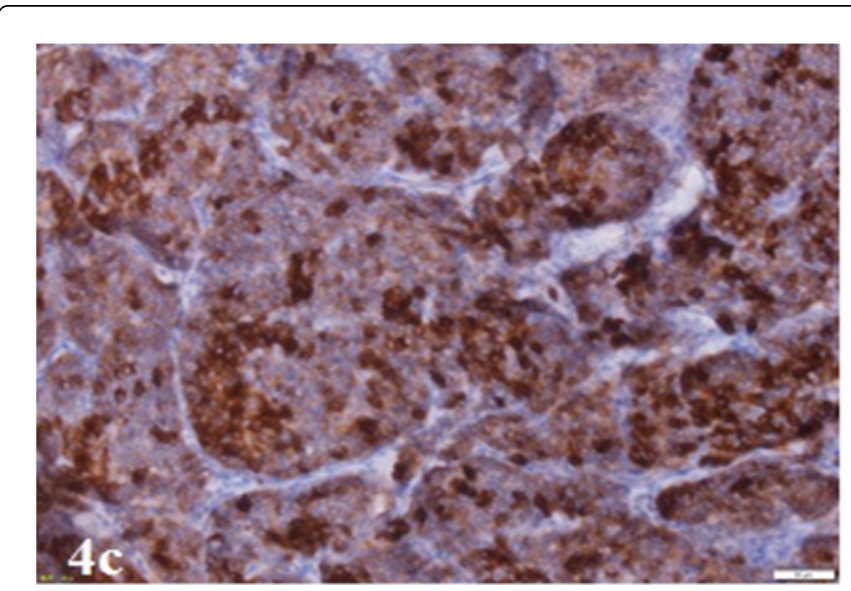

Figure 4c: No mitosis or necrosis was present (H\&E stain X200). Immunostaining showing a tumor with diffusely and strongly positive chromogranin-A.

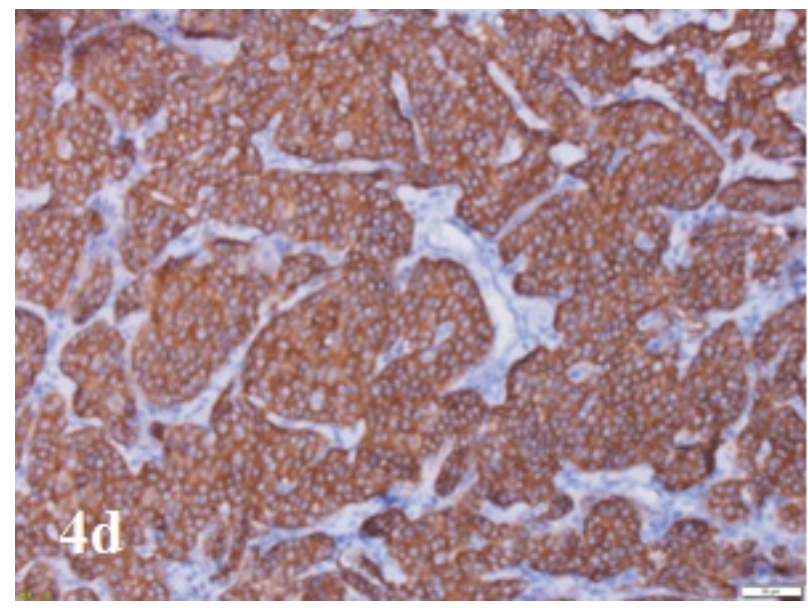

Figure 4d: Immunostaining showing a tumor with diffusely and strongly positive synaptophysin.

\section{Discussion}

Chronic cough has been defined as a "cough (as the only symptom) lasting for $>8$ weeks and with a cause that is not apparent by physical examination and routine testing such as CXR and spirometry" [1]. In the Hokuriku region of Japan, AC, CVA, and SBS are major causes of chronic cough [2].

Because both AC and CVA present with non-productive cough and eosinophilic inflammation of the airways, it is difficult to distinguish $\mathrm{AC}$ and CVA in daily clinical practice. According to the Japanese Guidelines on Cough, "therapeutic diagnosis with bronchodilators" is useful for distinguishing between CVA and AC [1].

In CVA, the fundamental physiological feature is a heightened cough response to bronchoconstriction [3], and bronchoconstrictiontriggered cough usually responds well to bronchodilators such as beta-2 stimulants.
In contrast, in $\mathrm{AC}$, enhanced sensitivity of cough receptors in the airway superficial layer is the fundamental feature, and AC does not respond to bronchodilators. Therefore, it is possible to make a diagnosis of CVA if cough disappears or is alleviated following 1-2 weeks of treatment with a bronchodilator.

$\mathrm{AC}$ responds to histamine $\mathrm{H} 1$ antagonist and inhaled corticosteroid therapy. SBS presenting with productive cough manifests as chronic sinusitis and chronic neutrophilic inflammation of the lower airways such as chronic bronchitis, diffuse bronchiectasis, and diffuse panbronchiolitis [4]. We diagnosed AC in our patient because his bronchodilator-resistant cough was almost resolved with histamine $\mathrm{H} 1$ antagonist and inhaled corticosteroids.

In our case, his non-productive cough was resistant to 10-day bronchodilator therapy. Furthermore, cough was almost resolved by inhaled corticosteroids. The Japanese Guidelines on Cough list the differential diagnosis of prolonged and chronic non-productive cough (apart from AC and CVA) as follows: ACE inhibitor-induced cough, gastroesophageal reflux-associated cough, post-infectious persistent cough, pertussis, chlamydia infection, mycoplasma infection, eosinophilic bronchitis without asthma, laryngeal allergy, chronic interstitial pulmonary disease, psychogenic/habit cough, tracheobronchial tuberculosis, endobronchial foreign body, and endobronchial malignancy[1].

Chest CT is performed to distinguish among malignancy, tuberculosis, and interstitial pulmonary disease, which can be lifethreatening in some cases. We have showed that $11.5 \%$ of patients with chronic cough had dual cough causing disease in the Hokuriku region of Japan [2]. In our case, AC and endobronchial carcinoid were causes of chronic cough and therefore remaining cough with histamine H1 antagonist and inhaled corticosteroids completely relieved after the resection of endobronchial-typical carcinoid from the left main bronchus. In atopic cough, antitussive medication is generally stopped after the complete resolution of cough and so-called maintenance therapy is not recommended [1]. Thus, a final diagnosis of AC and endobronchial-typical carcinoid was given as the cause of the chronic non-productive cough.

Bronchopulmonary carcinoid tumors are rare, comprising 1\%-2\% of all bronchopulmonary tumors [5] and approximately 25\% of all carcinoid tumors [6]. Carcinoid tumors are classified according to their pathological features as either typical carcinoid (TC, $<2$ mitoses $/ 2 \mathrm{~mm}^{2}$ of viable tumor) or atypical carcinoid (ATC, 2-10 mitoses, necrosis, or architectural disruption) [7]. TC is 8-10 folds more frequent than ATC and is typically present in younger patients between the 4th and 6th decades of life [8-10]. Seventy-five percent of bronchopulmonary carcinoid tumors are located in the central airways [11]. The left main bronchus accounts for $8 \%$ of central tumors [12]. Most TCs occur in the central airway and often produce symptoms as a result of partial or complete bronchial obstruction or because of its high vascularity.

Symptoms of bronchopulmonary carcinoid tumors include cough, hemoptysis, wheezing, recurrent pulmonary infection, obstructive pneumonia, and/or atelectasis. Five percent of bronchopulmonary carcinoid tumors have a normal chest roentgenogram, as observed in our case. Even when respiratory symptoms are present, the diagnosis of bronchopulmonary carcinoid tumors is often delayed [13].

Several studies have demonstrated that lung cancer is the cause of chronic cough in $\leq 2 \%$ of cases. Among the differential diagnosis of chronic cough, the percentage of cases with endobronchial malignancy appears to be low; however, physicians should not eliminate the 
possibility that the cough is because of a neoplasm, particularly with refractory cough cases.

Recently, a large-scale cohort study using the Internet to investigate the prevalence of cough in the general population in Japan revealed a prevalence of chronic cough of $>2 \%$ ( $>2.5$ million individuals). Cough has a negative effect on the quality of life [14]. We need to determine the differential diagnosis of the causative disease causing the cough with medical history taking and physical and clinical examinations. The appropriate differential diagnosis leads to a disease-specific therapy, for example, histamine $\mathrm{H} 1$ antagonist for $\mathrm{AC}$ or bronchodilator for CVA. If disease-specific therapy for treating the suspected cough-causing disease is insufficient, then we should be cognizant of the existence or coexistence of another underlying disease.

\section{References}

1. Kohno S, Ishida T, Uchida Y (2006) The Japanese Respiratory Society guidelines for management of cough. Respirology 4: S135-86.

2. Fujimura M, Abo M, Ogawa $H$ (2005) Importance of atopic cough, cough variant asthma and sinobronchial syndrome as causes of chronic cough in the Hokuriku area of Japan. Respirology 10: 201-207.

3. Ohkura N, Fujimura M, Nakade Y, Okazaki A, Katayama N (2012) Heightened cough response to bronchoconstriction in cough variant asthma. Respirology 17: 964-948.

4. Fujimura M, Ogawa H, Yasui M, Matsuda T (2000) Eosinophilic tracheobronchitis and airway cough hypersensitivity in chronic nonproductive cough. Clin Exp Allergy 30: 41-47.
5. Hage R, de la Rivière AB, Seldenrijk CA, van den Bosch JM (2003) Update in pulmonary carcinoid tumors: a review article. Ann Surg Oncol 10: 697-704.

6. Godwin JD (1975) Carcinoid tumors. An analysis of 2,837 cases. Cancer 36: 560-569.

7. Travis WD, Rush W, Flieder DB (1998) Survival analysis of 200 pulmonary neuroendocrine tumors with clarification of criteria for atypical carcinoid and its separation from typical carcinoid. Am J Surg Pathol 22: 934-944.

8. Hassan MM, Phan A, Li D, Dagohoy CG, Leary C, et al. (2008) Risk factors associated with neuroendocrine tumors: A U.S.-based casecontrol study. Int J Cancer 123: 867-873.

9. Soga J, Yakuwa Y (1999) Bronchopulmonary carcinoids: An analysis of 1,875 reported cases with special reference to a comparison between typical carcinoids and atypical varieties. Ann Thorac Cardiovasc Surg 5: $211-219$

10. Fink G, Krelbaum T, Yellin A (2001) Pulmonary carcinoid: presentation, diagnosis, and outcome in 142 cases in Israel and review of 640 cases from the literature. Chest 119: 1647-1651

11. Skuladottir H, Hirsch FR, Hansen HH, Olsen JH (2002) Pulmonary neuroendocrine tumors: incidence and prognosis of histological subtypes. A population-based study in Denmark. Lung Cancer 37: 127-135.

12. Detterbeck FC (2010) Management of carcinoid tumors. Ann Thorac Surg 89: 998-1005.

13. Kaif JT, Kayser G, Ruf J, Passlick B (2015) The diagnosis and treatment of bronchopulmonary carcinoid. Dtsch Arztebl Int 112: 479-485.

14. Fujimura M (2012) Frequency of persistent cough and trends in seeking medical care and treatment-results of an internet survey. Allergol Int 61: $573-581$. 